J. Acoust. Soc. Jpn. (E) 12, 5 (1991)

\section{Observation of the sound field in transient radiation from a concave piston source using time-transition pattern}

Keywords: Concave transducer, Impulsive ultrasound field, Time-transition pattern, Direct wave, Edge wave

PACS Number: 43. 35. Yb

\section{Kazuhiko Imano, Yasuo Yoshida, and Daitaro Okuyama \\ Department of Electronic Engineering, Mining College, Akita University, 1-1, Tegata gakuennmachi, Akita, 010 Japan (Received 4 February 1991)}

\section{Introduction}

The concave ultrasonic transducer is frequently used in equipment for ultrasonic diagnosis or nondestructive inspection to enhance the lateral resolution. In most of this equipment, the concave transducer is driven by a pulse or sinusoidal pulses. Impulsive ultrasound has recently also been used to enhance the time resolution. The sound field properties of the ultrasonic transducer are of prime importance in the evaluation of its performance and in its design. Although much analytical study on the sound field has been done, ${ }^{1,2)}$ reports of experimental study ${ }^{3)}$ have been rare except for the optical (e.g. Schlieren) method. ${ }^{4)}$

The authors recently reported a method of observing the transient field utilizing a hydrophone with scanning mode and a brightness modulation method. ${ }^{5)}$ According to the results, the transient field of the piston source is composed of two components: one is the direct wave (DW) radiated from the surface of the transducer and the other is the edge wave (EW) emitted from the transducers perimeter.

In this article, the impulsive sound field of the concave transducer is visualized by the time-transition pattern developed by the authors. The unique features of the impulsive ultrasound field of the concave transducer are demonstrated through experiments.

\section{Impulsive sound field of the concave transducer}

The sound field in transient radiation from a concave transducer may be divided into three regions as shown in Fig. 1.6) In region 1, both direct wave (DW) and edge wave (EW) exist, and DW arrives at the field point earlier than EW, that is, $t_{\mathrm{d}}\left(=l_{\mathrm{d}} / v\right)<t_{\mathrm{e}}\left(=l_{\mathrm{e}} / v\right)$, where $l_{\mathrm{d}}$ is the length of the leg, which is normal to the surface of the transducer, from a field point, and $l_{\mathrm{e}}$ is the minimum distance between edge of the transducer and a field point. And $v$ is the sound velocity of the medium. In region 2, only EW exists and there is no DW. In region 3, both DW and EW exist and EW arrives at the field point earlier than DW, that is $t_{\mathrm{d}}>t_{\mathrm{e}}$. In this region, DW has the opposite polarity of the sound pressure in region 1 .

The impulsive ultrasound of the concave transducer was then observed in the three regions described above to justify the hypothesis.

\section{Experimental results}

We applied the time-transition pattern as a tool for observation. The method of developing this timetransition pattern has been detailed by the authors. ${ }^{5)}$ The concave transducer used here is made of PZT ceramics and is baffled in a bakelite board. The diameter and radius of curvature are $50 \mathrm{~mm}$ and 75 $\mathrm{mm}$, respectively. The transducer was driven by a step voltage of $50 \mathrm{~V}$ having a rise time of $10 \mathrm{~ns}$. In order to discriminate the primary wave from the reverberation components, a thick transducer $(26 \mathrm{~mm}$ thick at the center) was used. Transducer and hydrophone were set in a water tank filled with degassed water. The hydrophone was scanned parallel to $\mathrm{A}-\mathrm{A}^{\prime}$ keeping the distance $H$ as indicated in Fig. 1.

Figure 2(a), (b) and (c) is the time-transition pattern of the sound field in transient radiation from the concave transducer for $H=15 \mathrm{~mm}, 75 \mathrm{~mm}$ and $90 \mathrm{~mm}$, respectively. The bright and dark parts of the field pattern correspond respectively to the negative and positive sound pressures. The components labeled $\mathrm{b} 1, \mathrm{~b} 2, \ldots$ are the reverberation components in the transducer. The usual $A$-mode (time-amplitude) waveforms of Fig. 2(a), (b) and (c), which is obtained on the axis of transducer, are indicated in Fig. 3 (a), (b) and (c), respectively. In the time-transition pattern, the vertical axis represents location of the hydrophone and the horizontal axis represents the time. Figure 2 (a), (b) and (c) reflects the following conditions: (a) the hydrophone was scanned in regions 1 and 2, that is, nearer the side of the transducer than the focal point; (b) the hydrophone was scanned just across the focal point; and (c) the hydrophone was scanned in regions 2 and 3 , which is far from the focal point. It is clear in each figure that the impulsive sound field of the concave transducer is composed of the direct wave and edge wave, similar to the plane transducer. DW is radiated from the surface of the transducer and EW is emitted from the perimeter. From the viewpoint of polarity of the sound pressure (referred to hereafter as polarity), DW always has the opposite polarity of EW in both regions 1 and 3. It is also apparent that polarity of DW is reversed before and behind the focal point. 
This inversion effect of polarity around the focal point corresponds to the phase inversion around the focal point in the steady state field. ${ }^{7)}$

Experimental results revealed the following: 1) in

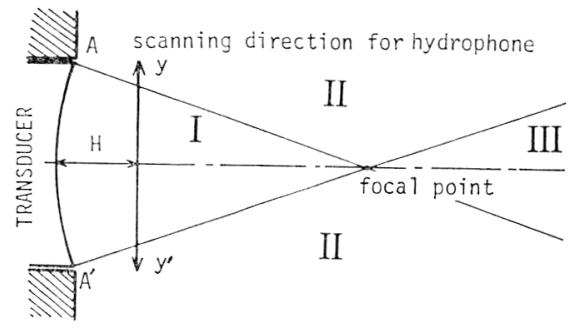

Fig. 1 Explanatory diagram of the sound field in transient radiation from a concave transducer.

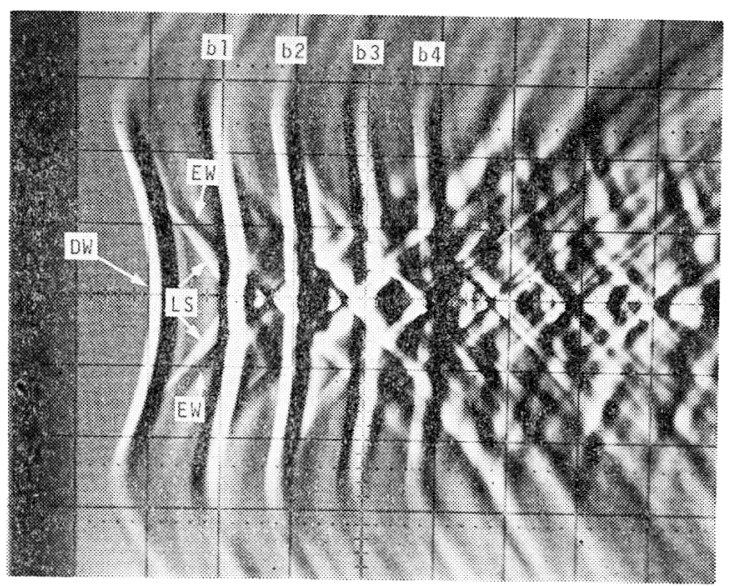

(a)

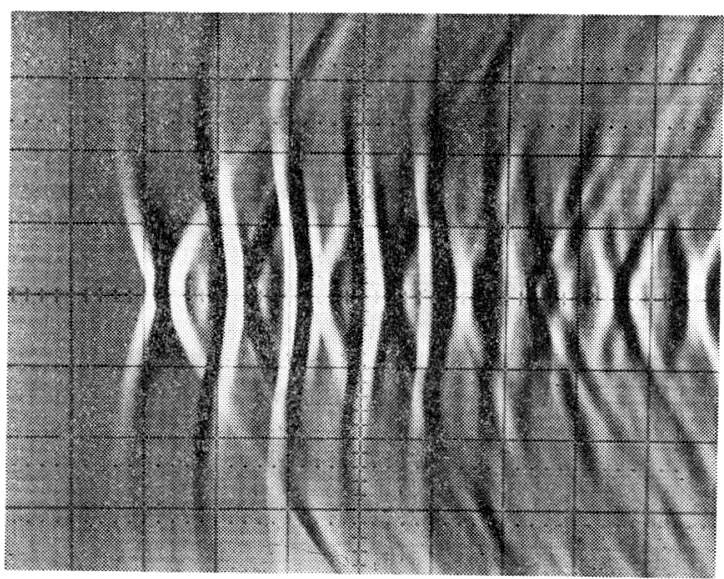

(b) region $1, \mathrm{DW}$ is a spherical convergence wave, 2) DW becomes a plane wave around the focal point and 3) DW is finally changed into a spherical diffused sound with the reversed polarity in region 1 . The phase inversion effect in the steady state field, in which the phase of the sound wave changes by $\pi / 2$ in both region 1 and 3 and total phase change becomes $\pi$, is easily explained from the experimental results.

In Fig. 2 (a), a wave component other than DW and $\mathrm{EW}$ is seen (denoted by LS in the figure). This wave component is also observed in a plane transducer; and its wavefront varies with the shape of the transducer surface, and it is therefore thought to be a leaky surface wave from the transducer. It is difficult by usual observation methods, for example, by time-amplitude display such as Fig. 3, to distinguish the EW or LS component from other components, so that the timetransition pattern is very effective for investigation of the sound field.

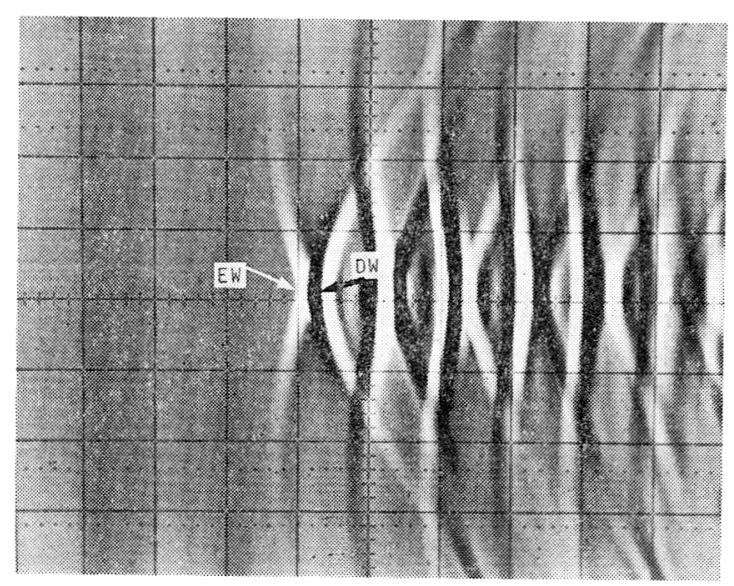

(c)

Fig. 2 Time-transition pattern of the impulsive ultrasound from a concave transducer. (a) $H=15 \mathrm{~mm}$, (b) $H=75 \mathrm{~mm}$ and (c) $H=90 \mathrm{~mm}$. The components $\mathrm{b} 1, \mathrm{~b} 2, \ldots$ are the reverberation components in the transducer. Horizontal scale (time): $5 \mu \mathrm{s} /$ div., vertical (position of hydrophone) scale: $1 \mathrm{~cm} /$ div. Sweeping trigger signal is delayed by $40 \mu \mathrm{s}$ in both (b) and (c). 


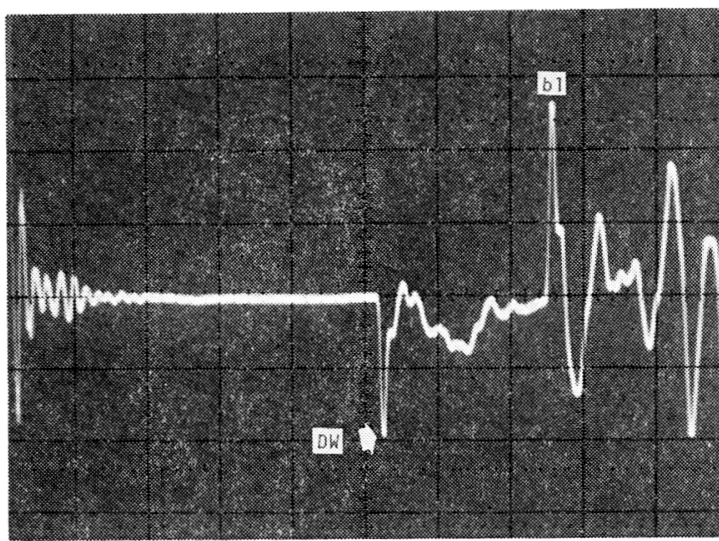

(a)

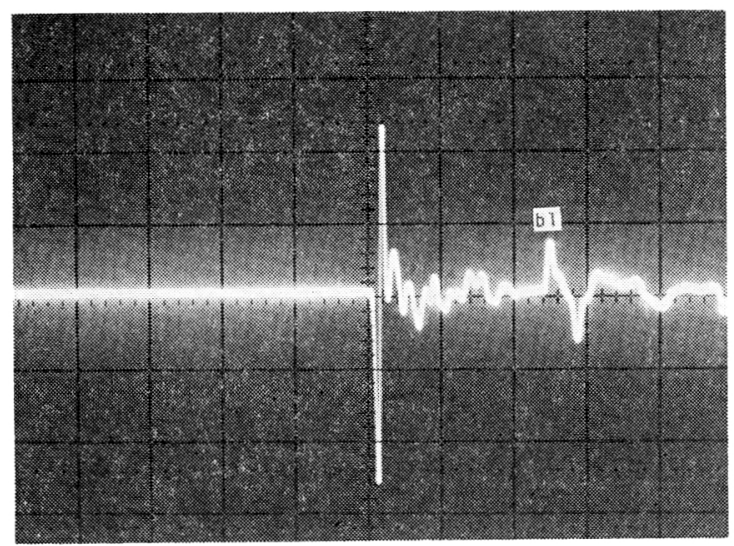

(b)

\section{Conclusion}

The impulsive ultrasound field from a concave transducer observed by the time-transition pattern was described. Components of this sound field and these of the distribution including polarity of sound pressure were clearly observed. These results thus provide a means of designing or evaluating a transducer for ultrasonic imaging and ultrasonic instrumentation.

We hope in future to observe the sound field of other type of transducer by various exciting method for the further investigation of the transducer performance.

\section{References}

1) F. Oberhettinger, "On the transient solutions of the baffled piston problem," J. Res. NBS, 65B, 1-6 (1961).

2) P. R. Stepannishen, "The time-dependent force and radiation impedance on a piston in a infinite

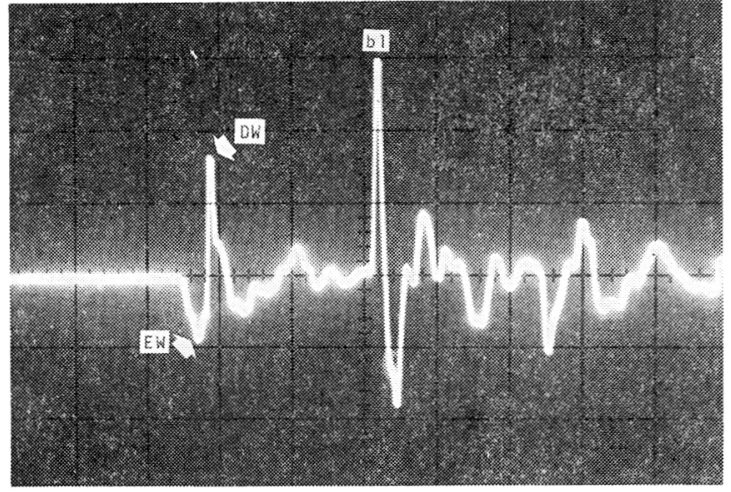

(c)

Fig. 3 Corresponding time-amplitude (pressure) display (A-mode) of Fig. 2. The hydrophone was located on the axis of the concave transducer. (a) $H=15 \mathrm{~mm}$, (b) $H=75 \mathrm{~mm}$ (focal point) and (c) $H=90 \mathrm{~mm}$. Time scale: $2 \mu \mathrm{s} / \mathrm{div}$. voltage scale: (a) $50 \mathrm{mV} /$ div., (b) $0.5 \mathrm{~V} /$ div. and (c) $0.2 \mathrm{~V} /$ div. Sweeping trigger signal is delayed by $40 \mu \mathrm{s}$ and $55 \mu \mathrm{s}$ in (b) and (c), respectively. From these figures, EW and LS components can not be distinguish from other (DW or reverberation) components. planar baffle," J. Acoust. Soc. Am. 49, 841-849 (1974).

3) A. J. Hayman and J. P. Weight, "Transmission and reception of short ultrasonic pulses by circular and square transducers," J. Acoust. Soc. Am. 66, 945-951 (1979).

4) K. Negishi and H. U. Li, "A model experiment of wave propagation in acoustic microscope," Jpn. J. Appl. Phys. S-27-1, 203-205 (1988).

5) K. Imano, Y. Yoshida, and D. Okuyama, "A method of observing the sound field in transient radiation from a piston source," J. Acoust. Soc. Jpn. 12, 233-235 (1991).

6) D. Okuyama, K. Imano, Y. Yoshida, H. Uemura, and Y. Sato, "The sound field in transient radiation of a concave transducer," IEICE Tech. Rep. EA-81-33, 49-54 (1981).

7) H. T. O'Neil, "Theory of focussing radiators," J. Acoust. Soc. Am. 44, 1310-1318 (1949). 